\title{
Critical appraisal of tools and methodologies for studies of cascading failures in coupled critical infrastructure systems \\ DOI:
}

10.1109/EUROCON.2017.8011182

\section{Document Version}

Accepted author manuscript

Link to publication record in Manchester Research Explorer

Citation for published version (APA):

Chen, Y., \& Milanovic, J. V. (2017). Critical appraisal of tools and methodologies for studies of cascading failures in coupled critical infrastructure systems. In IEEE EUROCON 2017-17th International Conference on Smart

Technologies https://doi.org/10.1109/EUROCON.2017.8011182

Published in:

IEEE EUROCON 2017 -17th International Conference on Smart Technologies

\section{Citing this paper}

Please note that where the full-text provided on Manchester Research Explorer is the Author Accepted Manuscript or Proof version this may differ from the final Published version. If citing, it is advised that you check and use the publisher's definitive version.

\section{General rights}

Copyright and moral rights for the publications made accessible in the Research Explorer are retained by the authors and/or other copyright owners and it is a condition of accessing publications that users recognise and abide by the legal requirements associated with these rights.

\section{Takedown policy}

If you believe that this document breaches copyright please refer to the University of Manchester's Takedown Procedures [http://man.ac.uk/04Y6Bo] or contact uml.scholarlycommunications@manchester.ac.uk providing relevant details, so we can investigate your claim.

\section{OPEN ACCESS}




\title{
Critical Appraisal of Tools and Methodologies for Studies of Cascading Failures in Coupled Critical Infrastructure Systems
}

\author{
Yushi Chen*, Jovica V.Milanović \\ Electrical Energy and Power Group \\ University of Manchester \\ Manchester, United Kingdom \\ *yushi.chen@postgrad.manchester.ac.uk
}

\begin{abstract}
Integration of critical infrastructures contributes to an increasing complexity and heterogeneity of their interdependencies, which results in a more detailed analysis process with emphasis on a certain aspect of the interactions such as cascading effects rather than the overarching system modelling and simulation. When external disturbances like natural disasters and intentional attacks take place, induced cascading failures are more likely to happen in large scale than they used to be. Their propagation paths and time-dependent behaviours are largely unknown to the area researchers and post-disaster consequences are rather difficult to quantify. This paper is presenting critical appraisal of existing methodologies for modelling cascading failures in interconnected systems. General explanations of the most widely used methodologies are firstly illustrated with the emphasis on their advantages and disadvantages and with the aim of choosing appropriate modelling and simulation methods for study of cascading failures in coupled electric power grids and telecommunication systems.
\end{abstract}

Keywords - cascading failures; modelling and simulation; infrastructure interdependencies

\section{INTRODUCTION}

Critical infrastructures (CIs) such as electric power system (EPS), transportation system, water distribution network and communication system (CS) can be no longer analysed alone as their interdependencies exert noticeable influences during both normal and abnormal operating periods. Rinaldi [1] summaries interdependencies into four classifications which are characterised as physical, cyber, geographical and logical. CIs with physical interdependencies rely on the material outputs of each other. Cyber interdependencies refer to crosssector influences caused by exchanging information with CS. Geographical interdependencies result from spatial adjacency and logical interdependencies are usually presented as financial connections, legal and political impacts. In the coupled model of EPS and CS, physical and cyber interdependencies are the major subjects to capture.

EPS consists of generators, transmission networks, distribution networks and consumptions of end users, which makes it bulk in size and diffused in resource allocations. But nowadays the system has an even more complex topological structure due to the tendency of distributed generations and smart communications [2]. Among large varieties of communication techniques, those that can provide services with high confidentiality and stable availability are vastly used for EPS. Their bandwidth and latency requirements are restrictive. Protection information within substations should be received no later than 0.004 seconds and the restriction for external transmission is 0.008 to 0.012 seconds [3]. From the view of embedded applications, modern EPS and CS express their integrated relationships typically as telecommunication system embedded in EPS like Supervisory Control and Data Acquisition (SCADA), and electricity sources for CS units.

Cascading failures that involve multiple CIs are extremely rare to happen but it becomes more frequent in recent years. As for long term impacts, large scales of people are affected and economic losses are huge. But during the short period after the triggering events, failures are spreading among CIs through their interdependencies in a considerable speed. In other words, interdependencies are the paths of their propagation and the only way to mitigate CI risks is to predict how cascading failures will perform given a certain context. This leads to three major tasks to model cascading failures: risk identification (RI) risk impact assessment (RIA) and risk prioritisation (RP) [4]. $\mathrm{RI}$ means to detect the potential risks in CIs contributed from other CIs through their interdependencies and identify the affected nodes and edges or risk propagation paths. RIA is to estimate the effects of the triggered events and to quantify the consequences with certain parameters. RP is to figure out the most vulnerable node or link that exposes CIs with largest risks All three tasks focus on the CI behaviours during a short period between initial event and the moment external mitigation measures are taken.

To start researching in analysing interdependent CIs, types of interdependencies, characteristics of research CIs, timevarying behaviours of CIs under vulnerabilities and specific tasks to achieve are the main aspects to take into account, which also applies to choosing appropriate methods as a beginning. Therefore this paper is to compare different methods that have been applied for modelling and simulation of CI interdependencies based on the aforementioned criteria. Focus is on selecting suitable methods with reference to a few comprehensive reviews, to discover the hidden patterns of cascading failures happened in the interactive system that consists of EPS and CS. Since only two CIs are considered, the descriptions are expected to be realistic in reappearing the scale and complexity of the systems.

\section{Modelling AND SimUlation METHODS FOR} ANALYSING INFRASTRUCTURE INTERDEPENDENCIES

In the following section, seven modelling and simulation methods developed for studying CI interdependencies are introduced and discussed. The discussion focuses mainly on explaining the methodology rationales, strengths, weaknesses and representative applications. 


\section{A. Agent Based Modelling}

Agent based modelling (ABM) has been applied in many areas due to its detailed illustrations of the system behaviours. In this model, the fundamental element "agent" is intended to provide certain service and governed by a series of what-if rules and pre-defined attributes [5]. The agents can therefore learn from their contexts and respond accordingly [6]. Based on this operating mechanism, agents are able to capture the real-world movements and interactions realistically, including time evolving behaviour between CIs that could be simulated by the mutual reactions of agents if sufficient input data are available. Typical attributes include name, coordinates, behaviour rules, memory, resources, decision making scenario and rules that modify the behaviour rules [7].

ABM has many advantages in modelling interdependent CIs. Its major feature, threshold based rules allow it to include nonlinearities and external contexts such as natural disasters, political laws or security protocols into the system modelling. All types of interdependencies can be expressed and embedded into the configurations of agents. The major downside of ABM is high computational cost (up to 50 hours for each simulation [8]) due to its low level abstraction. Abstraction level is an index that tells how generalised the description is. In other words, sufficient details are required for each agent and large amounts of data are desired, making the method unscalable for large system modelling. Experts on the incorporated CIs are also needed to provide assumptions on attributes and rules in case of scant input data. Therefore it is not practical to utilise sole ABM for modelling CIs with hundreds of buses especially when they are coupled. Integration with other methods is a reasonable way for optimisation

FedABMS [9] combines ABM with federated simulation method to exploit both advantages. For interdependent CIs, agents that describe the behaviours of their elements are reusing the existing simulation models to build up agent attributes and threshold rules. Parallel analysis is therefore allowed and computational cost reduces. In [10], anticipation game theory is incorporated with ABM to simulate the impacts of Distributed Denial of Service (DDoS) attacks on CIs in the form of cyber interdependencies. In the model, the major players, defenders and attackers, are represented as agents in the game and they interact based on the rules derived from DDoS graph. Reference [11] uses ABM to provide reductionistic construction for the framework called mixed holistic reductionist approach which couples high level description with low level description. A tool for analysing cascading failures named CISIA is introduced. In conclusion the $A B M$ is suitable for hybrid integration.

\section{B. Network Based Modelling}

Nodes and edges are the key elements of Network based modelling (NBM). Nodes represent the components of CIs and edges represent the links between two nodes. By using graph theory, NBM provides a straightforward presentation of the topological structure and flow patterns within CIs, which is a good feature for modelling cascading effects in a large system. Physical interdependencies such as electricity transmissions are unidirectional in the model while cyber interdependencies are marked as bidirectional links. Impacts of cascading failures can be statistically shown with indicators. Depending on whether nodes and edges are illustrated in detail, NBM can be divided as topology based modelling and flow based modelling [12].

1) Topology Based Modelling: In this methodology, topologies are the only criteria for the studies of interdependencies among CIs and each node has only two states, working or failed [13]. Few data are needed and computational cost is rather low. However, it models dynamic events in a discrete way. Complex network theory (CNT) is a typical topology-based modelling (TBM) approach. It has been extended from modelling standalone CI to analysing infrastructure interdependencies [14]. Structural indices such as node degree and betweenness centrality are used to identify critical nodes that are exposed to vulnerability [8]. The nature of the method is the shortest path theorem which calculates the minimised sum of the weights of paths between two nodes [15] If the sum is larger the vulnerability is higher.

In TBM, the way to express one-to-one interdependencies can be quite different in order to explore the complex percolation scenarios [12]. Take a coupled system with infrastructure $A$ and $B$ for explanation. In [14] each node in $A$ is interdependent on one and only one node in $B$ and all nodes in $B$ have their corresponding nodes in $A$; in [16] a fraction of nodes in $A$ are dependent on some of nodes in $B$ and similarly a fraction of nodes in $\mathrm{B}$ are dependent on some nodes in $A$; in [8] only certain part of a critical node is actually triggering indirect effects so more detailed abstraction is needed for these types of nodes but one-to-one relationship instead of one-to-fraction or fraction-to-fraction is used.

2) Flow Based Modelling: Unlike TBM, flow based modelling (FBM) provides more detailed and realistic interpretations (e.g., the services nodes offer) of the infrastructure behaviours. Therefore dedicated flow based methods for different CIs are used with coordinators involved.

In [17], flow based models developed for analysing power system and gas system are combined to study interdependencies. The simulation results are surprisingly different from those by topology-based modelling. The used flow based method for power system is ORNL-PSerc-Alaska (OPA) model [18], where nodes represent different buses with active power injection vectors and edges are electric lines with active power flow vectors. Basic information like different notations for generation nodes and load nodes, maximum power limits at the buses, and transmission capacity limits of the edges can be embedded [19]. Three models, reliability block diagrams (RBD), stochastic activity networks (SAN), and network reliability analyser (NRA), are used together in [20] to support the quantification of mutual impacts among telecommunication network, telecommunication emergency power supply and power distribution grid respectively, under the circumstance that critical communication links are disconnected due to faults. Each model presents the flows of different media (logic, data and power) in different ways.

During the applications of FBM, longer CPU time is needed since specific flow patterns of the CIs are highlighted with dedicated flow models and background knowledge concerning the existing modelling methods for single infrastructure is required for researchers.

\section{Economic Based Modelling}

To address the lack of mathematical simulation methods, some economic based models are extended for coupled infrastructure studies. In the following sections, two models that are well suited into the system behaviour analysis and large system modelling are introduced. 
1) Inoperability Input-output Modelling: Inoperability input-output modelling (IIM) is a continuous modelling method for multi-sector analysis, descended from Leontief Input-Output model [21]. With the major function of analysing short-term dynamics, IIM uses data collected at the normal conditions to simulate the situation of CIs during the immediate period after the initial event happens. The nature of IIM is that a system should have a balance between inputs and outputs based on the concept of inoperability, which refers to the timevarying unavailability of a working infrastructure to provide its assigned services [22].

$$
x=A x+c \leftrightarrow\left\{x_{i}=\sum_{j} a_{i j} x_{j}+c_{i}\right\} \forall i
$$

In (1), $x_{i}$ is the total risk of inoperability that infrastructure $i$ could possibly experience; $a_{i j}$ is the probability of inoperability of infrastructure $i$ given the state of infrastructure $j ; c_{i}$ represents the inherent risk of inoperability contained in infrastructure $i$ [12]. In other words, $\sum_{j} a_{i j} x_{j}$ is the sum of indirect effects that result from other infrastructures. For analysis in economic aspects, large input-output data sets are available in the Bureau of Economic Analysis (BEA) [23], based on which a technical matrix for nearly 500 sectors has been generated to analyse the interdependencies between U.S. economy and workforce [22]. But for other infrastructure modelling, this type of data is scant and can only be collected from literature.

By upgrading the formulation with a dynamic variable that represents the infrastructure resilience to the induced disturbances, IIM is extended to be Dynamic Inoperability Input-output modelling (DIIM) which would be a suitable method to model continuous cascading failures. In [24], fuzzy logic is combined with DIIM to overcome the limitation of deficient data sources. To be precise, technical coefficients are expressed as fuzzy numbers and the inoperability of each sector is presented as a fuzzy variable. The ambiguity range of each parameter is based on the expert experience. In [25], ABM is involved with DIIM as AB-DIIM and in this model, the media that propagates among different interdependent sectors is not the risk of inoperability but services performed by different agents. In other words, inoperability is redefined as an abstract parameter that tells the overall interactions of the agents inside a sector.

In general, IIM is a useful risk-based tool that can be easily combined with other methods. Its computational cost is small since simulation is not undertaken but modelling calculations. However, it has some limitations due to the linear expression. Over pessimistic results are likely to get because IIM assumes that no response will be made and self-healing ability as well as infrastructure resilience are neglected [26]. Its way of illustrating system mechanism is highly abstractive without component details, which calls for reductionist methods like ABM.

2) Computable General Equilibrium: Computable general equilibrium (CGE) method is an progressive extension of IIM as it overcomes the weakness of IIM in modelling nonlinearities [27]. A comprehensive view of economic aspects is offered by considering the post-disaster changing prices [28]. Many key features of IIM are reserved for CGE modelling such as modelling interdependent behaviours of multi-sectors. While IIM analyses short-term dynamics, CGE is regarded as a method for assessing medium to long term impacts. As for practical applications, CGE is widely used for studying mainly macroeconomic issues especially in developing countries where reliable time-series data are not adequate and significant policy changes are more likely to take place [29]. But applying CGE method to analyse multiple types of infrastructure interdependencies is not so mature.

The method originated from the concept of general equilibrium. General equilibrium refers to a balanced state of the overall economy which results from the interactions among several markets [30]. In CGE model, producer and customer are the two major players who interact to reach general equilibrium by means of supply, demand and prices [31]. In the infrastructure model, electricity and information are regarded as commodities. In simple terms, electricity is produced by generators and sold to customers while information is generated by monitoring devices and sold to regional control centres. As the actual interdependencies are of higher complexity, a set of equations together with corresponding data sets are used to represent the infrastructure behaviours when equilibrium of the overall system is reached [32].

Practical problems emerge from calibrating production and selling functions since it is not easy to find adequate relevant data [33]. In addition, CGE is based on over optimised assumptions that every element of CIs are in optimal conditions and that the economy is in balance before perturbations [27]. Actual economic costs are claimed to be underestimated due to the involvement of short-term input substitution (it is assume that goods can be produced by substituted materials) [34].

In [35], case studies on 9/11 terrorist attack are conducted using CGE to determine the indirect effects. A few disequilibria are incorporated in analysis for assessing direct impacts. Economic resilience is measured as parameters at different aggregation level. Reference [31] proposed a generalised modelling framework combining multilayer infrastructure networks (MIN) and spatial computable general equilibrium (SCGE) method. With spatial characteristics taken into account, a three dimensional framework of MIN is constructed to model various types of interdependencies simultaneously and captures the discrete decision-making behaviours of producers, customers and transport agents. The framework is extended in [36] and interdependencies are studied in dynamic and disequilibrium contexts.

\section{Petri Nets}

Petri nets (PN) are a promising tool developed for describing the concurrent processes of a complex system with multiple components. It is featured as a graphical tool similar to flow chart and block diagram and also a mathematical tool to quantify system behaviours with matrix calculations [37]. Similar to NBM, PN are able to incorporate the topological characteristics of complex CIs by applying the concept of conditions and events [38] to clarify the sequential movements in the network. Each petri net describes a single process with key elements of places, transitions, arcs and tokens. Take a low level infrastructure model for illustration: Places refer to the conditions of infrastructure components; transitions are the events that change the component states; unidirectional arcs connect places with transitions and show the process direction; tokens represent the material subjects that are exchanged during the events between or among the particular places. In [12], [39], PNs are referred with the ability of modelling only physical interdependencies while the opposite opinions also exist [8]. 
By using PN for analysis, both high level and low level descriptions can be realised. Continuous and discrete timedependent simulations are also achievable. From this point of view, PN are beneficial for analysing cascading failures and tracing dissemination of disruptions that happen without involving CS. The size of PN however, could easily expand to an explosive level for large system modelling and thereby the computational cost will be very high. So far, there has been very little research on addressing the side effects of growing size of the model.

Coloured Petri Nets (CPN) [40], are used with appropriate mathematical structures to study the spreading mechanism of the prevailing disruption. Based on the preliminaries of CPN theory, an integrated method named Timed Coloured Petri Nets (TCPN) is applied in [41], which adds time intervals and token values as additional attributes to the transitions. TCPN are used to capture the cause-effect interdependencies between sea ports and their supply chains in a continuous time scale under the framework of several patterns that arise commonly in supply chains. Reference [42] have proposed an integrated PN based method that combines SAN and Stochastic Well-formed Network (SWN) to quantify the failure consequences in the coupled system of EPS and CS. For CS infrastructure modelling, SWN is used as a highly abstractive method that depicts the attack or failure scenarios. SAN is selected as a supplement to describe the impacts of a DDoS attack to EPS. The results are presented as various attributes such as the percentage of mean power demand that is not met in the required time interval.

\section{E. Bayesian Network}

Bayesian network (BN) is a useful tool based on Bayes' Theorem. The conditional probabilities of events $A$ and $B$ satisfy the relationship expressed in (2) [43].

$$
P(A \mid B) P(B)=P(B \mid A) P(A)
$$

As is mentioned in [44], $\mathrm{BN}$ is able to provide a acyclic graphical solution for infrastructure interdependency analysis. In this methodology, nodes and edges are used as the main elements as well where nodes represent random variables and edges are unidirectional arcs connect one node to another [45]. Each node has its parent node(s) and they are assumed to be only dependent on parents not their grandparents. Edges are always pointed from parent nodes to their children, which mean the spreading relationships could not go back to the nodes that are passed through. In the coupled system of CIs, nodes refer to the services that are offered by infrastructure elements and the quality of their service is dependent on some other services (parent nodes). If a fault event occurs at a node, its edges will point at the children and change their states.

However, complete topologies of CIs are not usually needed since $\mathrm{BN}$ is only focusing on the part of coupled system that is influenced under a certain scenario [46]. So after identifying the affected services and devices, functional attributes are only assigned to affected nodes. Besides from these, each node has a conditional probability table (CPT) for probabilistic calculation [47]. Increase complexity of topology will lead to increase size of CPT for a node as each CPT contains the probabilities of the node at different states given the condition of its parents. In real cases the behaviours of nodes are usually assessed with ranks according to expertise or literature [46] so that probabilities could be estimated. This is still a difficult task and the size of CPT can be much larger.
Once the CPTs for involving nodes are available, the risks and interdependencies of CIs could be analysed.

An integrated method, Dynamic Bayesian Network (DBN) has been used in [48], [49] to analyse CI interdependencies. DBN introduced a concept of intertime-slice links to represent the time-varying interdependencies, i.e., interactions in the time dimension are separated from the interactions at the static time slices. Risk prediction is proposed as one of the achievable tasks if DBN is used. The idea is to update CPTs in each time slice by learning from historical data and then use them to estimate the most possible conditions of nodes in each time frame. The feasibility of this approach though has not yet been confirmed. DBN has also been used [50] for making decisions about extreme events as it can handle probabilistic events and provide advice on the feasibility of mitigation measures.

In summary, BN is a user-friendly method as it does not require much expertise in the domain of modelling rationale. It is also scalable for large system modelling especially for datadriven tasks. During the application, data such as conditional probabilities of any two nodes (parent and children) are required. If these data are not available in literature or database, an intensive expert work is needed and bias will follow. These problems have seldom been addressed as the up-to-data techniques of BN are still subject of early stage research.

\section{COMPARISONS}

As discussed in the introduction, to select a suitable method one should consider not only usability but also other casedependent criteria. Given this perspective, five criteria are selected for grading performances of the methods and another five criteria are used to show the distinctiveness of each approach. Data acquisition is not included as one of the criteria for comparisons as it is one of the major unsolved challenges of most methods. To visualise the comparisons, radar diagram Fig. 1 and Table I. are used.

Comparative criteria for Fig.1 are selected based on [8], [12], [39] to include both fundamental requirements and specialised performance. Since the case studies on testing different methods have not yet been realised, judgements on grades are depending on the overall perceptions from previous experienced researchers in [4], [8], [12], [31], [33], [34], [39], [44], [51]-[54]. According to their semantic descriptions, it is relatively easy to find the best and worst methods based on a criterion, which are assigned with 5 and 1 . The rest are then compared to them and roughly ranked with the grades. Fig. 1 shows differences in the following aspects:

\section{1) Practicality:}

a) Computational speed: It is a metric to record how fast the modelling and simulation process will be. Methods that can deploy large systems are not necessarily faster than
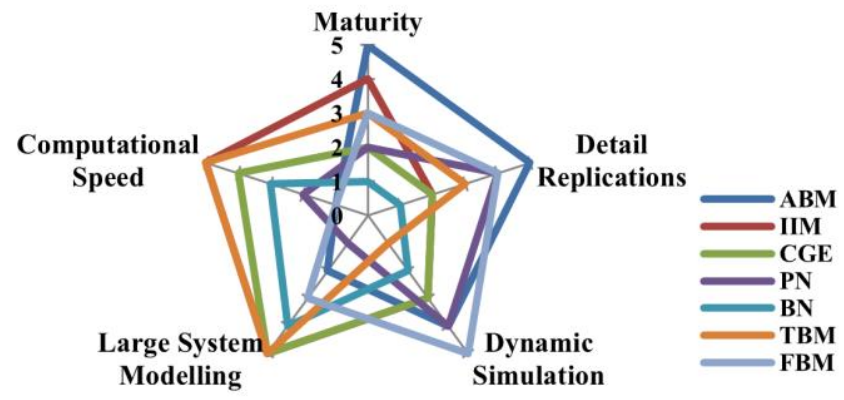

Fig. 1. Comparative diagram of seven methods based on five critical criteria 
TABLE I. OTHER CRUTIAL FEATURES OF DIFFERENT METHODS

\begin{tabular}{|c|c|c|c|c|c|}
\hline Approach & $\begin{array}{c}\text { Types of } \\
\text { Interdependencies [39] }\end{array}$ & Major Advantages & Tasks $[4]$ & Tools & $\begin{array}{c}\text { Abstraction } \\
\text { level }\end{array}$ \\
\hline $\mathrm{ABM}$ & $\mathrm{P}, \mathrm{C}, \mathrm{G}, \mathrm{L}$ & Realistic interactions & RI & Swarm, Repast and Netlogo & Low \\
\hline IIM & $\mathrm{P}, \mathrm{C}$ & Short term dynamics & RI, RIA & RIPS, ISA and REAcct [54] & High \\
\hline CGE & $\mathrm{P}, \mathrm{C}, \mathrm{G}, \mathrm{L}$ & Long term dynamics & RI, RIA & GEMPACK, GAM [4] & High \\
\hline $\mathrm{PN}$ & $\mathrm{P},(\mathrm{C})$ & Sequential events & RIA & Matlab, CPN Tools and Artifex [8] & High/low \\
\hline $\mathrm{BN}$ & $\mathrm{P}, \mathrm{C}, \mathrm{G}, \mathrm{L}$ & Conditional probabilities & RI, RP & Hugin, Netica and Analytica [50] & Low \\
\hline TBM & $\mathrm{P}, \mathrm{C}, \mathrm{G}, \mathrm{L}$ & Topologies & RIA, RP & NetworkX, igraph, SNAP and MatlabBGL [8] & High/low \\
\hline FBM & $\mathrm{P}, \mathrm{C}, \mathrm{G}, \mathrm{L}$ & Flow patterns & RIA, RP & OPA and IRRIIS [4], [19] & Low \\
\hline
\end{tabular}

others since the difficulties of calculation algorithm and desired quantities of inputs could also influence the speed.

b) Maturity: It is used to describe the state-of-the-art of the methods. Number of relevant publications, number of available tools and major challenges to face are taken into accounts. If the core concepts are accepted broadly in the area and the major breakthroughs are made based on these standardised acknowledgements, the development of the method is regarded as mature.

\section{2) Cascading failure characteristics:}

a) Dynamic simulation: This is the major criterion for modelling cascading failures because the time-varying behaviours of involved CIs are the most attractive and useful phenomena to study for making recovery strategies. Continuous models worth 5 , discrete models are 1 . The possibility of enhancement represents the increment of grades.

b) Detail Replications: This metric assesses the ability of depicting not only disturbances but also normal conditions of the involved CIs. It does to some degree relate to the abstraction level since realistic description is achievable only if sufficient details are available. But low level abstraction sometimes does not require much information such as TBM.

c) Large system modelling: Typically a model with over 300 nodes is considered as large system while a model with nodes fewer than 100 is regarded to be small.

In the Table I. "P", "C", "G" and "L" refer to physical, cyber, geographical and logical interdependencies. Not all the available tools are listed but some common ones.

Given the criteria that are set up for evaluation, the ideal method for modelling and simulation of induced cascading failures between EPS and CS should be able to (1) analyse physical and cyber interdependencies; (2) model the continuous time-dependent behaviours; (3) capture topological characteristics; (4) model large system; (5) describe at certain level of abstraction to conserve fidelity; (6) have relatively low computational costs; (7) realise all three tasks, RI, RIA and RP. Obviously there is no such method that could offer all of the advantages. Thereby a generalised option is to incorporate two or three methods together to fulfil different requirements. In Fig.1, it is indicated that the opposing differences among methods are mostly related to large system modelling and detail replications, or the different characteristics of the CI behaviours. So given this fact, two ways of coordinating different methods can be proposed. One is to combine a high level abstractive method with a low level one, such as IIM with ABM, high level TBM with BN. The other type is to model EPS and CS separately with different models and use a third model to connect them. For example, PN is a suitable method for EPS to replicate sequential events and to model its physical interdependencies with CS while ABM is appropriate for CS since human behaviours are involved as power system operations. The coordinator may be a dedicated framework or a master simulation platform.

Nevertheless the effectiveness of combination can only be judged after deploying the methods and tools. It is vital to be aware that these methods are different in many dimensions and more restrictive context requirements or assumptions may sometimes be made as premises for the incorporation.

\section{CONCLUSIONS}

Although there are many reviews on the mainstream methods of analysing infrastructure interdependencies, comparisons given defined contexts of coupled EPS and CS have not yet been made. With the aim of choosing appropriate methods for studying cross-sector cascading failures, thorough comparisons are conducted as a first step of the overall interdependency studies. Seven candidate methods for modelling and simulation of cascading effects in critical interconnected infrastructure systems are introduced as they all have some distinctive advantages in modelling coupled EPS and CS. Specific criteria are selected to compare and highlight their strengths and weaknesses for the specific purpose. General solutions are concluded as utilising different combinations of candidate methods to analyse the cascading failures in depth.

\section{REFERENCES}

[1] S. M. Rinaldi, "Modeling and simulating critical infrastructures and their interdependencies," 37th Annu. Hawaii Int. Conf. Syst. Sci. 2004. Proc., vol. 0 , no. C, pp. 1-8, 2004.

[2] R. Zimmerman, "Social implications of infrastructure network interactions," J. Urban Technol., vol. 8, no. 3, pp. 97-119, 2001.

[3] V. Chukwuka, M. H. Nazari, and S. Grijalva, "Enhancing communications reliability in prosumer-based smart grid using MIMO techniques," in 2016 Clemson University Power Systems Conference (PSC), 2016.

[4] G. Stergiopoulos, E. Vasilellis, G. Lykou, and D. Gritzalis, "Classification and comparison of critical infrastructure protection tools," J. Healthc. Prot. Manage., vol. 28, no. 2, pp. 101-13, 2016.

[5] T. Brown, W. Beyeler, and D. Barton, "Assessing infrastructure interdependencies: the challenge of risk analysis for complex adaptive systems," Int. J. Crit. Infrastructures, vol. 1, no. 1, pp. 108-117, 2004.

[6] C. G. Rieger, K. L. Moore, and T. L. Baldwin, "Resilient control systems: a multi-agent dynamic systems perspective," 2013.

[7] C. M. Macal and M. J. North, "Tutorial on agent-based modeling and simulation," Proc. 37th Conf. Winter Simul., pp. 2-15, 2005.

[8] J. Libardo and S. Torres, "Vulnerability , interdependencies and risk analysis of coupled infrastructures: power distribution network and ICT," University Grenoble Alpes, 2013.

[9] E. Casalicchio, E. Galli, and S. Tucci, "Federated agent-based modeling and simulation approach to study interdependencies in IT critical nfrastructures," Proc. - IEEE Int. Symp. Distrib. Simul. Real-Time Appl. DS-RT, pp. 31-38, 2007.

[10] R. Poisel, M. Rybnicek, and S. Tjoa, "Game-based simulation of Distributed Denial of Service (DDoS) attack and defense mechanisms of Critical Infrastructures," Proc. - Int. Conf. Adv. Inf. Netw. Appl. AINA, pp. 114-120, 2013.

[11] C. Foglietta, S. Panzieri, and F. Pascucci, "Identifying critical infrastructure clusters via spectral analysis," Stud. Comput. Intell., vol. 
565, pp. 223-235, 2015

[12] M. Ouyang, "Review on modeling and simulation of interdependent critical infrastructure systems," Reliab. Eng. Syst. Saf., vol. 121, pp. 4360, 2014.

[13] L. Cuadra, S. Salcedo-Sanz, J. Del Ser, S. Jiménez-Fernández, and Z. W. Geem, "A critical review of robustness in power grids using complex networks concepts," Energies, vol. 8, no. 9, pp. 9211-9265, 2015.

[14] S. V Buldyrev, R. Parshani, G. Paul, H. E. Stanley, and S. Havlin, "Catastrophic cascade of failures in interdependent networks," Nature, vol. 464, no. 7291, pp. 1025-1028, 2010.

[15] B. Wu, A. Tang, and J. Wu, "Modeling cascading failures in interdependent infrastructures under terrorist attacks," Reliab. Eng. Syst. Saf., vol. 147, pp. 1-8, 2016.

[16] R. Parshani, S. V. Buldyrev, and S. Havlin, "Interdependent networks: Reducing the coupling strength leads to a change from a first to second order percolation transition," Phys. Rev. Lett., vol. 105, no. 4, pp. 23-26, 2010.

[17] M. Ouyang and L. Dueñas-Osorio, "An approach to design interface topologies across interdependent urban infrastructure systems," Reliab. Eng. Syst. Saf., vol. 96, no. 11, pp. 1462-1473, 2011.

[18] I. Dobson et al., "An initial model for complex dynamics in electric power system blackouts," 36th Annu. Hawaii Int. Conf. Syst. Sci. 2003. Proc., vol. 0, no. January, pp. 318-326, 2003.

[19] S. Mei, F. He, X. Zhang, S. Wu, and G. Wang, "An improved OPA model and blackout risk assessment," IEEE Trans. Power Syst., vol. 24 no. 2, pp. 814-823, 2009.

[20] A. Bobbio et al., "Unavailability of critical SCADA communication links interconnecting a power grid and a Telco network," Reliab. Eng. Syst. Saf., vol. 95, no. 12, pp. 1345-1357, 2010.

[21] Y. Y. Haimes and P. Jiang, "leontief-based model of risk in complex interconnected infrastructrues," vol. 7, no. 1, pp. 1-12, 2001.

[22] Y. Y. Haimes, B. M. Horowitz, J. H. Lambert, J. Santos, C. Lian, and K. Crowther, "Inoperability input-output model for interdependent infrastructure sectors. I: theory and methodology," J. Infrastruct. Syst., vol. 11, no. 2, pp. 67-79, 2005.

[23] J. R. Santos, K. D. S. Yu, S. A. T. Pagsuyoin, and R. R. Tan, "Timevarying disaster recovery model for interdependent economic systems using Hybrid input-output and event tree analysis," Econ. Syst. Res., vol. 26, no. 1, pp. 60-80, 2014

[24] G. Oliva, S. Panzieri, and R. Setola, "Fuzzy dynamic input-output inoperability model," Int. J. Crit. Infrastruct. Prot., vol. 4, no. 3-4, pp. $165-175,2011$.

[25] G. Oliva, S. Panzieri, and R. Setola, "Agent-based input-output interdependency model," Int. J. Crit. Infrastruct. Prot., vol. 3, no. 2, pp. 76-82, 2010.

[26] R. M. Kolacinski, A. Kanchanaharuthai, and K. A. Loparo, "A general mathematical framework for power system security and control," IEEE 2011 EnergyTech, ENERGYTECH 2011, no. May, pp. 1-6, 2011.

[27] A. Rose, Rose, A., and A. Rose, "Input-output economics and computable general equilibrium models," Struct. Chang. Econ. Dyn., vol. 6, no. 3, pp. 295-304, 1995.

[28] S. Tsuchiya, H. Tatano, and N. Okada, "Economic loss assessment due to railroad and highway disruptions," Econ. Syst. Res., vol. 19, no. 2, pp. 147-162, 2007.

[29] P. D. Adams and B. R. Parmenter, Handbook of computable general equilibrium modeling, vol. 1. Elsevier, 2013.

[30] R. M. Starr, "Concept and history of general equilibrium theory," in General Equilibrium Theory: An Introduction, Cambridge University Press, 2011, pp. 3-40.

[31] P. Zhang and S. Peeta, "A generalized modeling framework to analyze interdependencies among infrastructure systems," Transp. Res. Part B Methodol., vol. 45, no. 3, pp. 553-579, 2011

[32] Z. Wu, E. Sherry, and J. Davis, "How economic analysis can inform the climate change policy debate: The case of Northern Ireland," EuroChoices, vol. 14, no. 1, pp. 42-47, 2015.

[33] S. Hasan and G. Foliente, "Modeling infrastructure system interdependencies and socioeconomic impacts of failure in extreme events: emerging R\&D challenges," Nat. Hazards, vol. 78, no. 3, pp. 2143-2168, 2015.

[34] S. Kelly, "Estimating economic loss from cascading infrastructure failure: a perspective on modelling interdependency," Infrastruct. Complex., vol. 2, no. 1, p. 7, 2015.

[35] A. Z. Rose, G. Oladosu, B. Lee, and G. B. Asay, "The economic impacts of the September 11 terrorist attacks: a computable general equilibrium analysis," Peace Econ. Peace Sci. Public Policy, vol. 15, no. 2, 2009.

[36] P. Zhang and S. Peeta, "Dynamic and disequilibrium analysis of interdependent infrastructure systems," Transp. Res. Part B Methodol., vol. 67, pp. 357-381, 2014.

[37] K. Salimifard and M. Wright, "Petri net-based modelling of workflow systems: An overview," Eur. J. Oper. Res., vol. 134, no. 3, pp. 664-676, 2001.

[38] T. Murata, "Petri nets: properties, analysis and applications," Proc. IEEE, vol. 77, no. 4, pp. 541-580, 1989.

[39] I. Eusgeld, D. Henzi, and W. Kröger, "Comparative evaluation of modeling and simulation techniques for interdependent critical infrastructures," 2008.

[40] S. H. Zegordi and H. Davarzani, "Developing a supply chain disruption analysis model: Application of colored Petri-nets," Expert Syst. Appl., vol. 39, no. 2, pp. 2102-2111, 2012.

[41] R. Liu, A. Kumar, and W. van der Aalst, "A formal modeling approach for supply chain event management," Decis. Support Syst., vol. 43, no. 3, pp. 761-778, 2007.

[42] M. Beccuti, S. Chiaradonna, F. Di Giandomenico, S. Donatelli, G. Dondossola, and G. Franceschinis, "Quantification of dependencies between electrical and information infrastructures," Int. J. Crit. Infrastruct. Prot., vol. 5, no. 1, pp. 14-27, 2012.

[43] A. Stuard and K. Ord, Kendalls advanced theory of statistics-volume I: distribution theory. ew York: Wiley, 2001.

[44] G. Satumtira and L. Dueñas-Osorio, "Synthesis of modeling and simulation methods on critical infrastructure interdependencies research," Sustain. Resilient Crit. Infrastruct. Syst. Simulation, Model. Intell. Eng., pp. 1-51, 2010.

[45] T. Koski and J. Noble, Bayesian networks: an introduction. John Wiley \& Sons, 2011.

[46] A. Di Giorgio and F. Liberati, "A Bayesian network-based approach to the critical infrastructure interdependencies analysis," IEEE Syst. J., vol. 6, no. 3, pp. 510-519, 2012.

[47] T. Schaberreiter, P. Bouvry, J. Roning, and D. Khadraoui, "Support tool for a Bayesian network based critical infrastructure risk model," in EVOLVE - A Bridge between Probability, Set Oriented Numerics, and Evolutionary Computation V, vol. 288, 2014, pp. 61-78.

[48] T. Schaberreiter, P. Bouvry, J. Röning, and D. Khadraoui, “A Bayesian network based critical infrastructure risk model," EVOLVE - A Bridg. between Probab. Set Oriented Numer. Evol. Comput. II, vol. 175, pp. 207-218, 2013.

[49] A. Di Giorgio and F. Liberati, "Interdependency modeling and analysis of critical infrastructures based on Dynamic Bayesian Networks," 2011 19th Mediterr. Conf. Control Autom., pp. 791-797, 2011.

[50] M. K. Jha and R. A. Keele, Using dynamic Bayesian networks for investigating the mpacts of extreme events. INTECH Open Access Publisher, 2012.

[51] T. Münzberg, M. Wiens, and F. Schultmann, "Dynamic-spatial vulnerability assessments: a methodical review for decision support in emergency planning for power outages," Procedia Eng., vol. 78, pp. 7887, 2014.

[52] N. Solomakhina, M. Watzke, F. Maréchal, S. Becher, S. Lamparter, and T. Hubauer, "Modeling and analysis techniques for multimodal utility networks," Energy Procedia, vol. 78, pp. 3397-3402, 2015.

[53] I. B. Utne, Henrik Hassel, and J. Johansson, "A brief overview of some methods and approaches for investigating interdependencies in critical infrastructures," pp. 1-11, 2013.

[54] E. Wiseman and N. Mrad, "Critical infrastructure protection and resilience literature survey: modeling and simulation," 2014. 